\title{
Using the Matlab Software for Music Arranger and Creation
}

\author{
Yanan Zhu ${ }^{1}$, Li Zhang, ${ }^{1, a}$ \\ ${ }^{1}$ School of Music, Tonghua Normal University, Tonghua, China \\ ${ }^{1}$ School of Physics, Tonghua Normal University, Tonghua, China \\ a dbcy9999@163.com \\ *corresponding author
}

Keywords: Music; Keyboard;Do Re Mi; Vibrations and Modes; Harmony and Intonation.

Abstract. Use computer software for arrangements, we are prepared to use the Matlab software, also known as matrix lab, one for algorithm development, data visualization, data analysis and numerical calculation of senior technical computing language and interactive environment. According to the basic knowledge of music theory, using the computer software programming realize simulation of arrangements, draw the hits "legend" and "Canon" of computer programming software, so as to better the application of computer software for editing the track simulation.

\section{Music}

What does $\sqrt[12]{2}$ have to do with music[1-5]?

In the theory of music,an octave is an interval with frequencies that range over a factor of two.In most Western music,an octave is divided into 12 semitones with equal frequency ratios.Since 12 semitones comprise a factor of 2,a single semitone is a factor of $\sqrt[12]{2}$.And because this quantity occurs so often in this chapter,let

$$
\sigma=\sqrt[12]{2}
$$

Our MATLAB programs use sigma $=2 \wedge(1 / 12)$

$$
=1.059463094359295
$$

Think of $\sigma$ as an important mathematical constant,like $\pi$ and $\phi$.

\section{KEYBOARD}

Our miniature piano keyboard with 25 keys.This keyboard has two octaves, with white keys labeled C D...G A B,plus another C key.Counting both white and black,there are twelves keys in each octave.The frequency of each key is semitone above or below it or the flat of the white above it.So the black key between $\mathrm{C}$ and $\mathrm{D}$ is both $\mathrm{C} \#$ and $\mathrm{D}^{b}$. There is no $\mathrm{E} \# / \mathrm{F}^{b}$ or $\mathrm{B} \# / \mathrm{C}^{b}$. 
A conventional full piano keyboard has 88 keys.Seven complete octaves account for $7 \times 12=84$ keys.There are three additional keys at the lower left and one additional key at the upper end.If the octaves are numbered 0 through 8,then a key letter followed by an octave number specifies a unique key.In this notation,two important keys are C4 and A4.The C4 key is near the center of the keyboard and so is also known as middle C.A piano is usually tuned so that the frequency of the A4 key is $440 \mathrm{~Hz}$.C4 is nine keys to the left of A4 so its frequency is

$$
\mathrm{C} 4=4400^{-9} \approx 261.6256 \mathrm{~Hz}
$$

Our EXM program pianoex uses C4 as the center of its 25 keys,so the number range is 12:122. The statement

Pianoex $(0)$

generates and plays the sound from a sine wave with frequency C4.The resulting visual display is shown in Figure 20.2.

This for loop plays the two octave chromatic scale formed from the 25 notes on our miniature keyboard.

$$
\begin{aligned}
& \text { for } n=-12: 12 \\
& \text { pianoex(n) } \\
& \text { end }
\end{aligned}
$$

\section{DO RE MI}

One of the first songs you learned to sing[6-13] was

\section{Do Re Mi Fa So La Ti Do}

If you start at C4,you would be singing the major scale in the key of C.This scale is played on a piano using only the white keys.The notes are not equally spaced.

Most of the steps skip over black keys and so are two semitones.But the steps between $\mathrm{Mi}$ and Fa and $\mathrm{Ti}$ and Do are the steps from $\mathrm{E}$ to $\mathrm{F}$ and $\mathrm{B}$ to $\mathrm{C}$.There are no intervening black keys and so these steps are only one semitone.In terms of $\sigma$,the C-major is

$$
\sigma^{0} \sigma^{2} \sigma^{4} \sigma^{7} \sigma^{9} \sigma^{11} \sigma^{12}
$$

You can play this scale on our miniature keyboard with

for $\mathrm{n}=\left[\begin{array}{llllllll}0 & 2 & 4 & 5 & 7 & 9 & 11 & 12\end{array}\right]$

$$
\text { pianoex(n) }
$$

end

The number of semitones between the notes is given by the vector diff ([0 $\left.\left.\begin{array}{lllllll}0 & 2 & 4 & 7 & 9 & 11 & 12\end{array}\right]\right)$

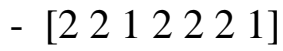

The sequence of frequencies in our most common scale is surprising. Why are there 8 notes in the C-major scale?Why don't the notes in the scale have uniform frequency ratios?For that matter,why is the octave divided into 12 semitones?The notes in "Do Re Me "are so familiar that we don't even ask ourselves these question. Are there mathematical explanations? I don't have definitive answers,but I can get some hints by looking at harmony,chords,and the ratios of small integers.

\section{VIBRATIONS AND MODES}

Musical[13] instruments create sound through the action of vibrating strings or vibrating columns of air that,in turn,produce vibrations in the body of the instrument and the surrounding air.Mathematically,vibrations can be modeled by weighted sums of characteristic functions known as modes or eigenfunctions. Different modes vibrate at different characteristic frequencies or eigenvalues.These frequencies are determined by physical parameters such as the length,thickness 
and tension in a string,or the geometry of the air cavity.Short,thin,tightly stretched string have high frequencies, while long,thick,loosely stretched string have low frequencies.

The simplest model is a one-dimensional vibrating string,held fixed at its ends.The units of the various physical parameters can be chosen so that the length of the string is $2 \pi$.The modes are then simply the functions

$$
\mathrm{v}^{k}(\mathrm{x})=\sin \mathrm{kx}^{1} \mathrm{k}=1,2, \ldots
$$

Each of these functions satisfy the fixed end point conditions

$$
\mathrm{V}^{k}(0)=\mathrm{V}^{k}(2 \pi)=0
$$

The time-dependent modal vibrations are

$\mathrm{v}^{k}(\mathrm{x}, \mathrm{t})=\sin \mathrm{kx} \sin \mathrm{kt}, \mathrm{k}=1,2, \ldots$

and the frequency is simply the integer k.(Two- and three-dimensional models are much more complicated.This one-dimensional model is all we need here.)

\section{CHORDS}

Chords are two or more notes played simultaneously.With a computer keyboard and mouse,we can't click on more than one key at a time.So chords are produced with pianoex by selecting the toggle switches labeled 1 through 12.The switch labeled 0 is always selected.

Figure 20.9 shows the visual output generated when pianoex plays a chord involving two adjacent white keys,in this case $\mathrm{C}$ and $\mathrm{D}$.You can see,and hear,the phenomenon known as beating. This occurs when tones with nearly equal frequencies alternate between additive reinforcement and subtractive cancellation.The relevant trig identity is

$$
\sin \mathrm{at}+\sin \mathrm{bt}=\sin \frac{a+b}{2} \mathrm{t} \cos \frac{a-b}{2} \mathrm{t}
$$

The sum of two notes is a note with the average of the two frequencies,modulated by a cosine term involving the difference of two frequencies. The players in an orchestra tune up by listening for beats between their instruments and a reference instrument.

The most important three-note chord(triad)is the $\mathrm{C}$ major fifth.If $\mathrm{C}$ is the lowest(root)note,the chord is C-E-G.In just intonation,the frequency ratio are

$$
1: \frac{5}{4}: \frac{3}{2}
$$

\section{CRIPPLE “ CANON” ON MATLAB SOFTWARE}

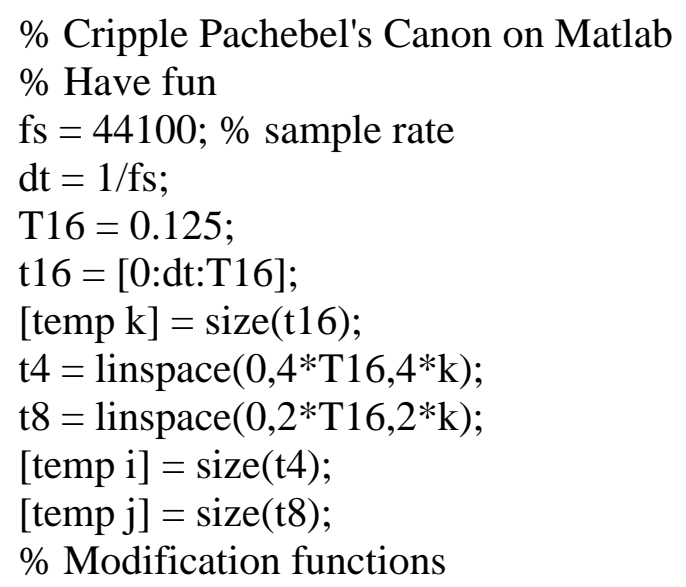




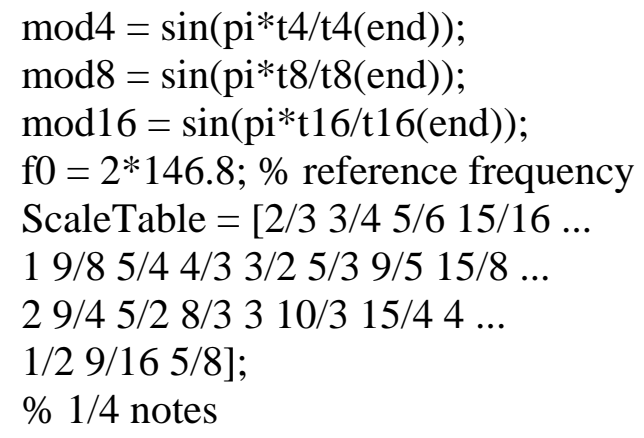

\section{CONCLUSION}

According to the music theory knowledge, we can use computer software programming to achieve the simulation, and draw the computer programming software, music and computer software, to better use computer software to simulate the music, and to display the complementary relationship between electronic instruments and traditional instruments, and provide a favorable technical means for digital simulation teaching and electronic vocal music experiment. Is conducive to the integration of various disciplines.

\section{References}

[1] K. Cascone, "The Aesthetics of Failure: 'Post-Digital' Tendencies in Contemporary Computer Music," Computer Music Journal, vol. 24, no. 4, pp. 12-18, Winter 2000

[2] J. Chadabe, "Remarks on Computer Music Culture," Computer Music Journal, vol. 24, no. 4, pp. 9-11, 2000.

[3] A. Collis, "Sounds of the System: the Emancipation of Noise in the Music of Carsten Nicolai," Organised Sound, vol. 13, no. 1, pp. 31-39, Feb. 2008.

[4] H. Cowell, "The Joys of Noise," in Audio Culture: Readings in Modern Music, C. Cox and D. Warner, Eds. New York: Continuum, 200

[5] J. T. Demers, Listening through the Noise: the Aesthetics of Experimental Electronic Music. Oxford, NY: Oxford University Press, 2010.

[6] A. Evens, Sound Ideas: Music, Machines, and Experience. Minneapolis, MN: University of Minnesota Press, 2005.

[7] G. Hainge, "Of Glitch and Men: The Place of the Human in the Successful Integration of Failure and Noise in the Digital Realm,” Communication Theory, vol. 17, no. 1, pp. 26-42, 2007.

[8] P. Hegarty, Noise/Music: a History. New York, NY: Continuum, 2007.

[9] C. Kelly, Cracked Media: the Sound of Malfunction. Cambridge, Mass.: MIT Press, 2009.

[10] B. Neill, "Pleasure Beats: Rhythm and the Aesthetics of Current Electronic Music," LMJ, vol. 12, pp. 3-6, 2002.

[11] T. Sangild, "Glitch- the Beauty of Malfunction," in Bad Music: the Music we Love to Hate, New York, NY: Routledge, 2004.

[12] P. Sherburne, “12k: Between Two Points,” Organised Sound, vol. 6, no. 3, pp. 171-176, Aug. 2002.

[13] A. Szepanski, “A Mille Plateaux Manifesto,” Organised Sound, vol. 6, no. 3, p. December 2001. 